Research Paper

\title{
Hydrogen Sulfide Inhibits Homocysteine-Induced Neuronal Senescence by Up-Regulation of SIRT1
}

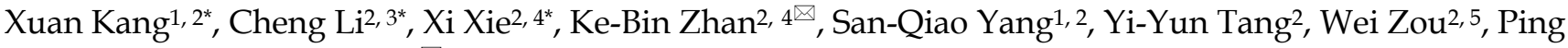 \\ Zhang 2, 5, Xiao-Qing Tang1, ${ }^{\bowtie}$ \\ 1. Institute of Neurology, the First Affiliated Hospital, University of South China, Hengyang, 42100, Hunan, P.R. China. \\ 2. Institute of Neuroscience, Hengyang Medical College, University of South China, Hengyang, 42100, Hunan, P.R. China. \\ 3. Department of Emergency, Affiliated Nanhua Hospital, University of South China, Hengyang, 421001, Hunan, P. R. China. \\ 4. Department of Neurology, the Second Affiliated Hospital, University of South China, Hengyang, 421001, Hunan, P.R. China. \\ 5. Department of Neurology, Affiliated Nanhua Hospital, University of South China, Hengyang, 421001, Hunan, P. R. China. \\ *Xuan Kang, Cheng Li and Xi Xie contributed equally to this work. \\ $\square$ Corresponding authors: Xiao-Qing Tang, Institute of Neuroscience, Hengyang Medical College, University of South China, 28 W Chang sheng Road, \\ Hengyang, 421001, Hunan, P. R. China, E-mail address: tangxq-usc@usc.edu.cn; tangxq-usc@qq.com and Ke-Bin Zhan, Department of Neurology, the Second \\ Affiliated Hospital, University of South China, Hengyang, 421001, Hunan, P.R. China, E-mail address: zhankebin@126.com; Zhankb-usc@usc.edu.cn \\ (c) The author(s). This is an open access article distributed under the terms of the Creative Commons Attribution License (https://creativecommons.org/licenses/by/4.0/). \\ See http://ivyspring.com/terms for full terms and conditions.
}

Received: 2019.07.21; Accepted: 2019.12.08; Published: 2020.01.17

\begin{abstract}
Homocysteine $(\mathrm{Hcy})$ accelerates neuronal senescence and induces age-related neurodegenerative diseases. Silence signal regulating factor 1 (SIRT1) prolongs lifespan and takes neuroprotective effects. We have previously demonstrated that hydrogen sulfide $\left(\mathrm{H}_{2} \mathrm{~S}\right)$ prevents Hcy-induced apoptosis of neuronal cells and has neuroprotective effect. In the present work, we aimed to investigate whether $\mathrm{H}_{2} \mathrm{~S}$ protects HT22 cells against Hcy-induced neuronal senescence and whether SIRT1 mediates this role of $\mathrm{H}_{2} \mathrm{~S}$. We found that Hcy induced cellular senescence in HT22 cells, as determined by $\beta$-galactosidase staining, expressions of P16INK4a, P21 CIPL, and trypan blue Staining, which are the markers of cellular senescence. However, sodium hydrosulfide ( $\mathrm{NaHS}$, the donor of $\mathrm{H}_{2} \mathrm{~S}$ ) significantly reversed Hcy-induced cellular senescence. Interestingly, NaHS not only up-regulated the expression of SIRT1 in HT22 cells but also reversed Hcy-downregulated the expression of SIRT1 in HT22 cells. Furthermore, we found that pretreatment with Sirtinol (an inhibitor of SIRT1) markedly reversed the protection of NaHS against Hcy-induced HT22 cells senescence and apoptosis. Our findings illustrated that $\mathrm{H}_{2} \mathrm{~S}$ protects $\mathrm{HT} 22$ cells against Hcy-induced senescence by up-regulating SIRT1.
\end{abstract}

Key words: cell senescence, homocysteine, hydrogen sulfide, SIRT1

\section{Introduction}

Homocysteine (Hcy) is a sulfur-containing nonprotein amino acid derive from methionine metabolism [1]. Hcy promotes neuronal degeneration and thus it contributes to age-related neurodegenerative diseases, such as dementia, Alzheimer's disease (AD), Parkinson's disease(PD), and stroke [2,3]. The emerging evidence suggests that a potentially important contributor to aging and age-related neurodegenerative diseases is cellular senescence [4], a process that imposes permanent proliferative arrest on cells responding to various stressors [5]. Therapeutic strategies that safely interfere with the detrimental effects of cellular senescence are gaining significant attention [4,6]. Interestingly, accumulating evidence showed that cellular senescence is causally implicated in Hcy-generated age-related diseases [7-9]. Thus, finding a new strategy to antagonize Hcyinduced neuronal senescence has important value in the prevention and treatment of age-related neurodegenerative diseases.

Hydrogen sulfide $\left(\mathrm{H}_{2} \mathrm{~S}\right)$ is a novel gaseous molecule with an extremely unpleasant odor. In the brain, $\mathrm{H}_{2} \mathrm{~S}$ is an important neuroprotective agent and has therapeutic potential in neurodegenerative diseases of aging, such as AD and PD [10,11]. Recently, $\mathrm{H}_{2} \mathrm{~S}$ was identifiedas a new approach to 
prolong lifespan [12]. $\mathrm{H}_{2} \mathrm{~S}$ inhibits the production of mitochondrial ROS [13], decreases oxidative stress $[14,15]$, and repairs DNA damage [16] to protect cells from senescence. Previous studies revealedthat $\mathrm{H}_{2} \mathrm{~S}$ protects endothelial cells against nicotinamide- [17] and $\mathrm{H}_{2} \mathrm{O}_{2-}$ [18] induced cellular senescence. Our previous study indicated that $\mathrm{H}_{2} \mathrm{~S}$ prevents Hcy-induced neurotoxicity $[19,20]$. In this study, we explored whether ameliorating Hcy-induced neuronal senescence contributes to the protection of $\mathrm{H}_{2} \mathrm{~S}$ against Hcy-induced neurotoxicity.

Silent mating type information regulator 2 homolog 1 (SIRT1) is a NAD+-dependent deacetylase of lysine residue of the target protein. SIRT1 extends lifespan [21] and improves cell tolerance to inhibit environmental stress [22,23]. Recently, it has shown that $\mathrm{H}_{2} \mathrm{~S}$ extends lifespan by activation of SIRT1 [12]. Furthermore, Our previous data demonstrated that $\mathrm{H}_{2} \mathrm{~S}$ up-regulates the expression of SIRT1 in PC12 cells [20]. Thus, we further investigated whether SIRT1 mediates the inhibitory role of $\mathrm{H}_{2} \mathrm{~S}$ in Hcy-induced neuronal senescence.

The present studies demonstrated the ability of $\mathrm{H}_{2} \mathrm{~S}$ to inhibit Hcy-induced senescence in HT-22 cells and identified the mediatory role of SIRT1 in the protective action of $\mathrm{H}_{2} \mathrm{~S}$ from Hcy-induced neuronal senescence. It provides novel strategy to prevent Hcy-induced neurotoxicity via inhibition of senescence-associated neuronal aging. Thus, our research identifies SIRT-1 as a potent therapeutic target and $\mathrm{H}_{2} \mathrm{~S}$ as a potent treatment for Hcy-related neurodegenerative diseases.

\section{Materials and Methods}

\section{Materials}

Sodium hydrosulfide, homocystein, sirtinol, dimethyl sulfoxide (DMSO), and propidium iodide (PI) were purchased from Sigma (St. Louis, MO, USA). Rh123 was supplied by Dojindo Molecular Technologies, Inc. (Rockvile, MD, USA). Specific monoclonal anti-SIRT1 antibody was obtained from Abcam (Hong Kong, China). Specific monoclonal antibodies of P16 $6^{\mathrm{INK} 4 a}$, P21 CIPL were purchased from OriGene Biotech Inc. (Burlingame, UK). Annexin V was bought from Nanjing Key GEN Biotech Co., Ltd. (Nanjing, China). DMEM medium, horse serum and fetal bovine serum were supplied by Gibico, BRL (Ground Island, NY, USA).

\section{Cell viability assay}

Cell viability was detected by Cell Counting Kit-8 (CCK-8) assay. HT22 Cells of logarithmic phase growth were seeded into a 96-well plate at a density of $1 \times 10^{4}$ cells/well. The cells were treated with 2.5, 5.0, $10.0 \mathrm{mM}$ Hcy, or/and 100, 200, $400 \mu \mathrm{mol} / \mathrm{L} \mathrm{NaHS}$ and routinely incubated for 24 hours, 48 hours, and 72 hours. After treatment, viable cells were stained with $5 \mu \mathrm{L}$ of CCK-8 solution (CCK-8, Sigma, USA) and incubated at $37^{\circ} \mathrm{C}$ for $4 \mathrm{~h}$. Absorbance was measured at $450 \mathrm{~nm}$ by a microplate reader (ELX-800, BIO-TEK, USA). The experiment was performed three times.

\section{$\boldsymbol{\beta}$-galactosidase (SA- $\boldsymbol{\beta}$-gal) staining assay}

Senescent cells were detected by the SA- $\beta$-gal staining. HT22 Cells were seeded into a 6-well plate at a density of $1 \times 10^{4}$ cells/well. After incubated with $2 \mathrm{ml} \mathrm{10 \%} \mathrm{Dulbecco's} \mathrm{Modified} \mathrm{Eagle} \mathrm{Medium} \mathrm{(DMEM,}$ Gibco, USA) for 24h, HT22 cells were treated with various concentrations of 2.5, 5.0, $10.0 \mathrm{mM} \mathrm{Hcy,}$ or/and 100, 200, $400 \mu \mathrm{mol} / \mathrm{L} \mathrm{NaHS}$ for $48 \mathrm{~h}$. The plates were stained daily using Senescence $\beta$-Galactosidase Staining Kit (Cell Signaling Technology, Danvers, MA, 9860) following the manufacturer's recommendations. All wells were counted in five randomized fields.

\section{Trypan blue Staining}

The cells were grew at concentration of $1 \times 10^{4}$ cells $/ \mathrm{ml}$ in 24 well-plates to $60-70 \%$ confluence by incubation for $24 \mathrm{~h}$, then treated with $2.5 \mathrm{mM}, 5.0 \mathrm{mM}$, $10 \mathrm{mM}$ Hcy or and 100, 200, $400 \mu \mathrm{mol} / \mathrm{L}$ NaHS for 24 h, $48 \mathrm{~h}, 72 \mathrm{~h}$. Floating and adhering cells were collected, washed once with PBS ( $\mathrm{pH}$ 7.4), centrifuged, suspended in 10\% DMEM and stained with $0.4 \%$ trypan blue (sigma, St. Louis, MO, USA) at room temperature. About $10 \mu \mathrm{l}$ of sample was loaded on hemocytometer chamber and numbers of blue cells and non-blue cells were counted under a light microscope (Mike audi, China). The results were expressed as percentage of the control.

\section{Western blotting}

Cells of logarithmic phase growth were plated in $50 \mathrm{ml}$ culture bottle. After incubated with DMEM for $24 \mathrm{~h}$, the cells were treated with Hcy or/ and NaHS at different concentrations for $48 \mathrm{~h}$. Then the treated cells were resuspended in $100 \mathrm{uM}$ cell lysis buffers and PMSF incubation on ice for $30 \mathrm{~min}$. The supernatant was collected after centrifuged at 12,000 $\mathrm{rpm}$ for $10 \mathrm{~min}$ at $4^{\circ} \mathrm{C}$. The protein concentrations were detected by BCA method (Solarbio, Beijing, China). Equal amounts of total protein extracts were electrophoresed through $10 \%$ or $12 \%$ SDS-PAGE gel, then transferred to polyvinylidene difluoride membranes (Solarbio, China). After blocked with TBST (50 mM Tris- $\mathrm{HCl}, \mathrm{pH} 7.4,0.15 \mathrm{M} \mathrm{NaCl}, 0.1 \%$ Tween-20) containing 5\% BSA (Sigma, USA) for $2 \mathrm{~h}$, the membranes were incubated with primary antibodies including monoclonal antibody for

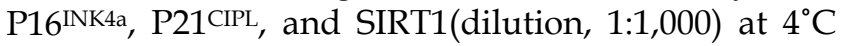
overnight. After washed with TBST for 5 min 5 times, 
the membranes were incubated with secondary antibodies including Goat anti-rabbit (Proteintech, USA) (dilution, 1: 5,000) at room temperature for $2 \mathrm{~h}$. After washed for three times with TBST for 3 times, the membranes were visualized with Western Blotting Chemiluminescence Reagent (Solarbio, Beijing, China), followed by apposition of the membranes with autoradiographic films (Kodak, China). The expression of $\beta$-actin for each sample was used as a control.

\section{Flow cytometry analysis}

HT22 cells of the log phase were grew in 6-well plates and treated with Hcy or NaHS for 48h. Cells

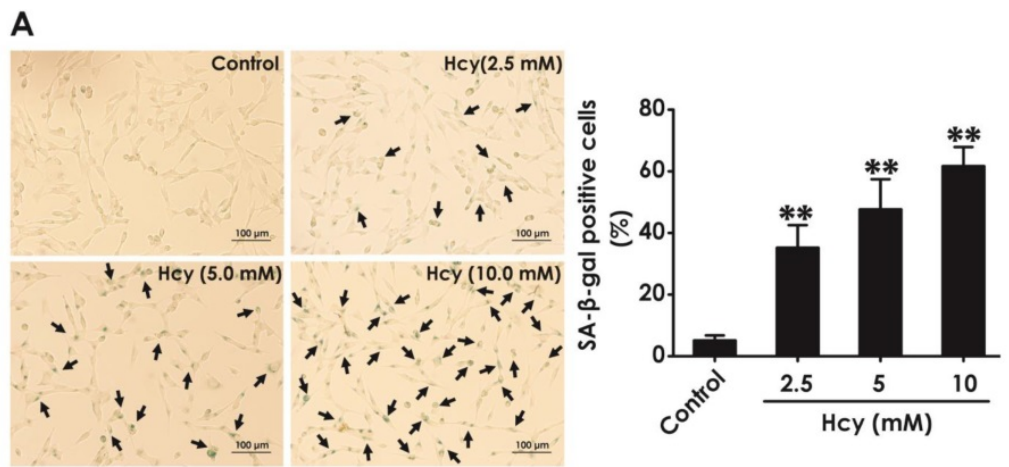

B
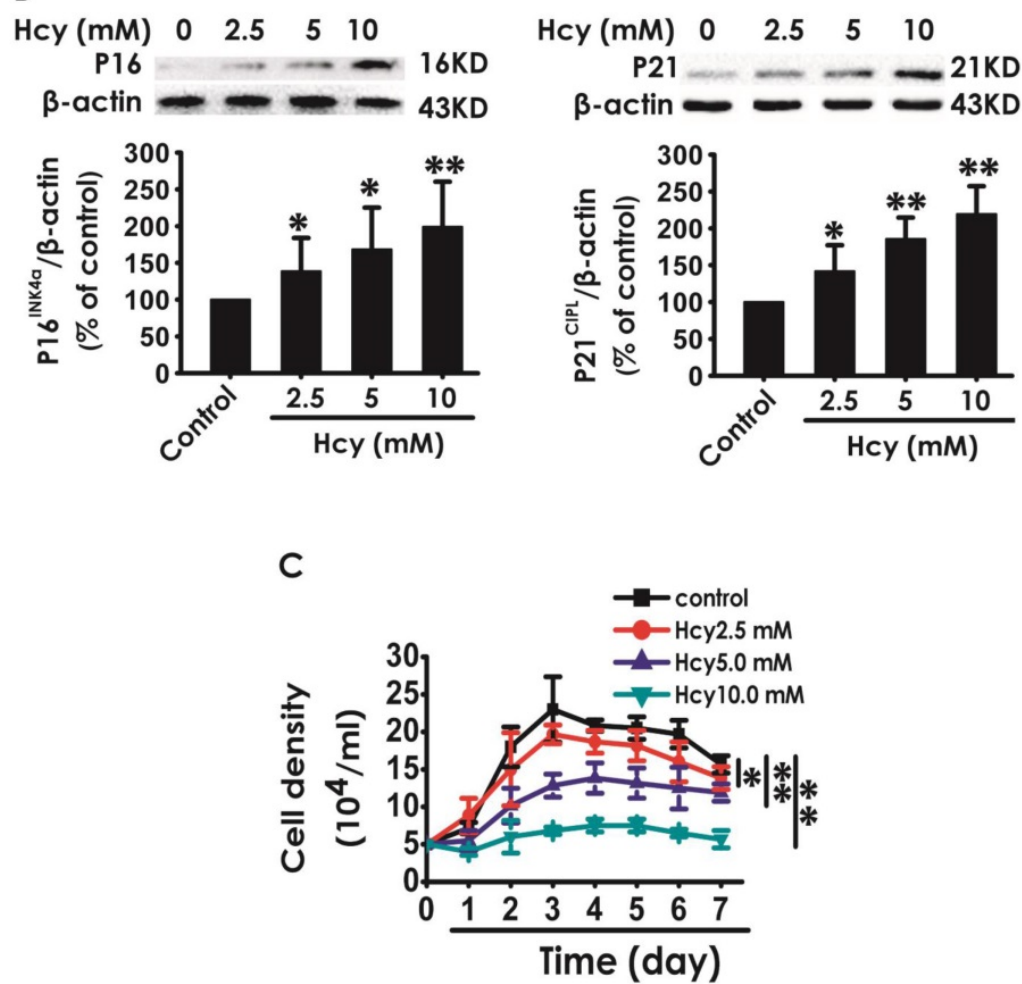

Figure 1. Effect of Hcy on the cellular senescence in HT22 cells. A, HT22 cells were stained with SA- $\beta$-gal and the SA- $\beta$-gal positive cell was quantitatively analyzed (magnification: $\times 10$; black arrows point the SA- $\beta$-gal staining positive cells). B, the expressions of P16INK4a and P2|CIPL in HT22 cells were measured by western blotting. C, the cell density was determined by trypan blue analysis and the growth curve for $7 \mathrm{~d}$ was drawn. Values are means $\pm \operatorname{SEM}(n=3)$. * $P<0.05$, **P<0.01, vs control group. were stained $50 \mu \mathrm{g} / \mathrm{ml}$ propidium iodide (PI) (Roche, Mannheim, Germany) and $10 \mu \mathrm{g} / \mathrm{ml}$ RNase A (Sigma, St. Louis, MO, USA). Percentages of cells existing within the various phases (G0/G1, S, G2/M) of the cell cycle were calculated by the mean fluorescence intensity.

\section{Statistical analysis}

Experiments were repeated at least three times. Values were expressed as mean \pm standard error of the mean (S.E.M.). All data were analyzed by SPSS version 21.0. Data were evaluated for statistical significance with using one-way analysis of variance (ANOVA) followed by LSD post hoc tests. $P<0.05$ was considered to indicate a statistically significant difference.

\section{Results}

\section{Hcy induced the cellular senescence in HT22 cells}

We first explored whether Hcy induces cellular senescence in HT22 cells. After treatment with Hcy $(2.5,5,10 \mathrm{mM})$ for $48 \mathrm{~h}$, the percentage of senescenceassociated beta-galactosidase (SA- $\beta-\mathrm{Gal})$ positive cells was increased (Fig. 1A), the expressions of P16 INK4a and P21CIPL were upregulated (Fig. 1B), and the cell density was decreased (Fig. 1C) in HT22 cells, which indicated that Hcy induces the cellular senescence in HT22 cells.

\section{$\mathrm{H}_{2} \mathrm{~S}$ prevented Hcy-induced cellular senescence in HT22 cells}

Next, we explored the effect of $\mathrm{H}_{2} \mathrm{~S}$ on Hcy-induced cellular senescence in HT22 cells. HT22 cells were pretreated with $\mathrm{NaHS}(100,200$, and $400 \mu \mathrm{M}$ ) for $30 \mathrm{~min}$ and then cotreated with $5 \mathrm{mM}$ Hcy for 48 h. We found that pretreatment of NaHS $(100,200$, or $400 \mathrm{mM})$ significantly decreased the percentage of SA- $\beta$-galpositive cells (Fig. 2A) and the expressions of P16 INK4a and P21CIPL (Fig. 2B), while increased the cell density (Fig. 2C) in Hcy-treated HT22 cells. These results demonstrated that $\mathrm{H}_{2} \mathrm{~S}$ prevented Hcyinduced cellular senescence in HT22 cells.

\section{NaHS upregulated the expression of SIRT1 in HT22 cells}

To explore the mediatory role of SIRT1 in the protection of H2S against Hcy-induced cellular senescence in HT22 cells, we first investigated the expression 
of SIRT1 in different treated HT22 cells. After the expression of SIRT1 in HT22 cells was markedly down-regulated by treatment with Hcy $(2.5,5.0,10.0$ $\mathrm{mM}$ ) for $48 \mathrm{~h}$ (Fig. 3A), while was up-regulated by treatment with NaHS (100, 200, and $400 \mu \mathrm{M})$ alone for 48 h (Fig. 3B). Furthermore, preteatment with NaHS
$(100,200$, and $400 \mu \mathrm{M})$ restored the expression of SIRT1 in Hcy-treated HT22 cells (Fig. 3C). These results suggest that NaHS not only upregulated the expression of SIRT1 in HT22 cells but also reversed the down-regulation of SIRT1 in Hcy-treated HT22 cells.

A
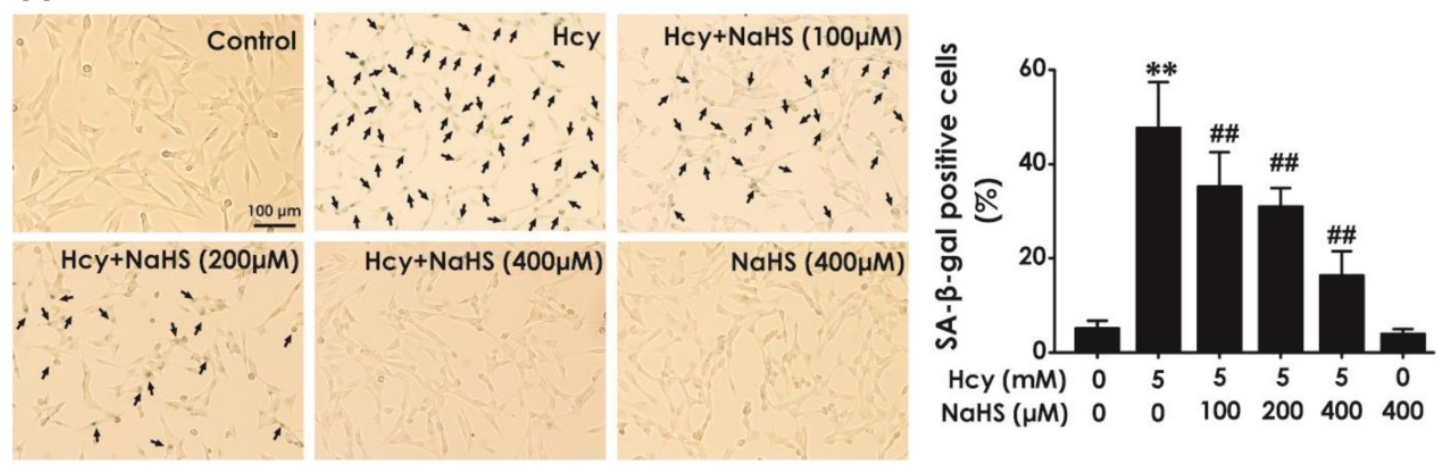

B

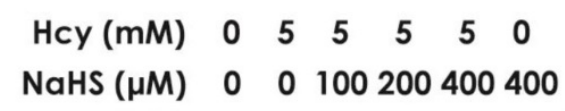

p16 - . 16KD

B-actin - - - - 43KD

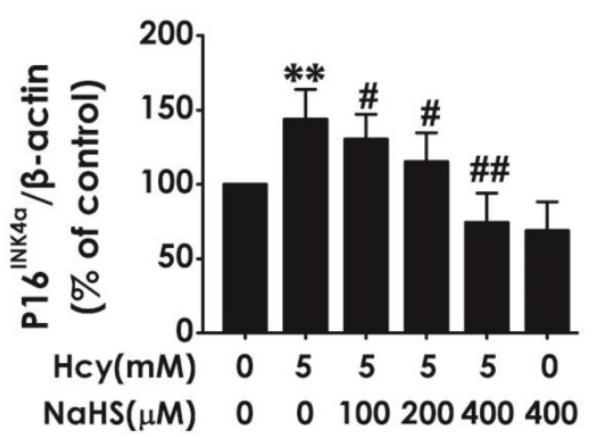

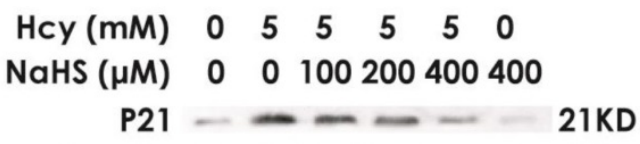

$\beta$-actin $-----43 K D$

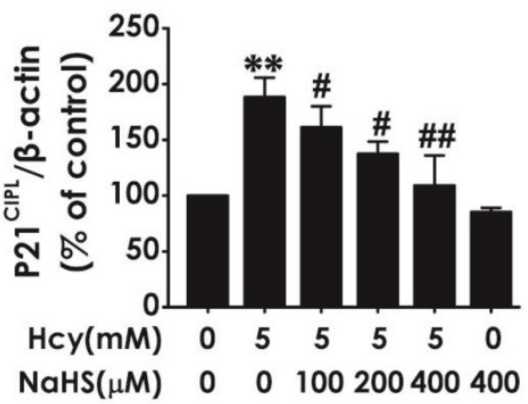

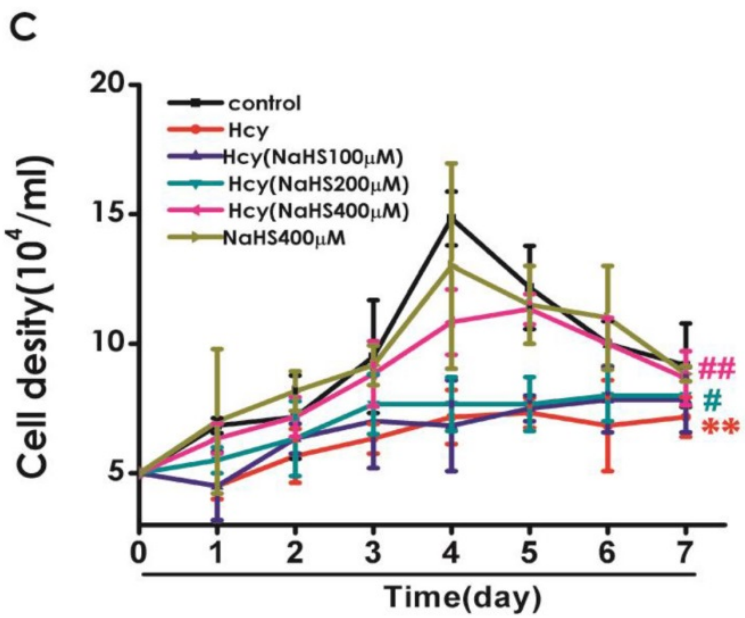

Figure 2. Effect of $\mathbf{H}_{2}$ S on Hcy-induced cellular senescence in HT22 cells. A, HT22 cells were stained with SA- $\beta$-gal and the SA- $\beta$-gal positive cell was quantitatively analyzed (magnification: $\times 10$; black arrows point the SA- $\beta$-gal staining positive cells). B, the expressions of P16INK4a and P21 CIPL in HT22 cells were measured by western blotting. $C$, the cell density was determined by trypan blue analysis and the growth curve for $7 \mathrm{~d}$ was drawn. Values are means $\pm S E M(n=3)$. $* * P<0.01, v s$ control group; $\# P<0.05$, $\# P<0.01$, vs Hcy-treated group. 
Sirtinol reversed the protection of $\mathrm{NaHS}$ against Hcy-induced cellular senescence in HT22 cells

To further confirm whether SIRT1 mediates the protection of NaHS against Hcy-induced cellular senescence in HT22 cells, we explored whether sirtinol, a specific inhibitor of SIRT1, reversed this protective role of NaHS. We found that pretreatment with sirtinol $(15 \mu \mathrm{M}$, for $30 \mathrm{~min})$ increased the percentage of SA- $\beta$-gal-positive cells (Fig. 4A) as well as the expressions of P16 ${ }^{\mathrm{INK} 4 \mathrm{a}}$, P21 $1^{\mathrm{CIPL}}$ (Fig. 4B), while decreased the cell density (Fig. 4C) in HT22 cells cotreated with $5 \mathrm{mM}$ Hcy and $400 \mu \mathrm{M}$ NaHS for $48 \mathrm{~h}$. These findings verified that sirtinol reverses the protection of NaHS against Hcy-induced cellular senescence in HT22 cells.

\section{Sirtinol reversed the protective effect of $\mathrm{H}_{2} \mathrm{~S}$ on Hcy-indcued neurotoxicity to HT22 cells}

We have previously demonstrated that $\mathrm{H}_{2} \mathrm{~S}$ inhibits Hcy-induced neurotoxicity. Therefore, we next investigated whether blockage of SIRT1 reverses the protective effect of $\mathrm{H}_{2} \mathrm{~S}$ against Hcy-induced neurotoxicity to HT22 cells. As shown in Fig. 5A,
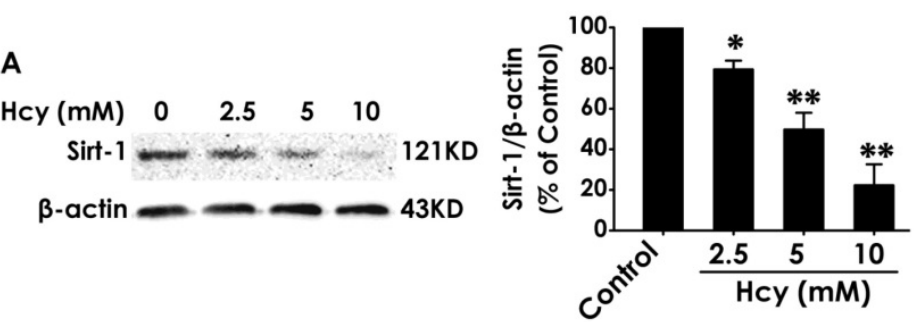

B
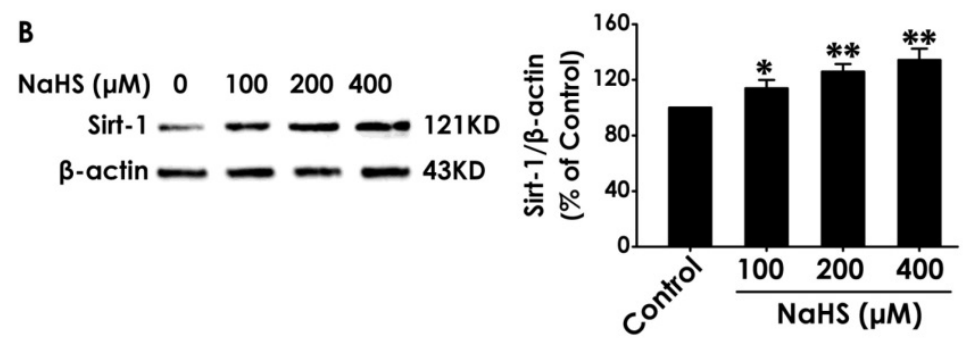
SIRT1 in HT22 cells treated with 48-h Hcy $(2.5,5.0,10.0 \mathrm{mM})$ alone (A), 48-h NaHS $(100,200,400 \mu \mathrm{mol} / \mathrm{L})$ alone (B), or 48- h cotreatment with Hcy (5.0) and NaHS $(100,200,400 \mu \mathrm{mol} / \mathrm{L})$ (C) were detected by western blotting. Values are means $\pm \operatorname{SEM}(n=3), * P<0.05$, $* * P<0.01$, vs control group; $\# P<0.05, \# P<0.01$, vs Hcy-treated alone group. pretreatment of NaHS $(100,200$, or $400 \mu \mathrm{M})$ significantly increased the cell viability of HT22 cells exposed to $5 \mathrm{mM}$ of Hcy for $48 \mathrm{~h}$, which indicated that NaHS inhibites Hcy-induced cytotoxicity to HT22 cells. However, pretreatment with sirtinol $(15 \mu \mathrm{M})$ markedly decreased the cell viability of HT22 cells cotreated with Hcy $(5 \mathrm{mM})$ and NaHS $(400 \mu \mathrm{M})$ (Fig. 5B), which indiccated that sirtinol reverses the protection of NaHS against Hcy-induced cytotoxicity to HT22 cells. In addition, pretreatment of NaHS (400 $\mu \mathrm{M}$, for $30 \mathrm{~min}$ ) significantly decreased the apoptosis in HT22 cells treated with $5 \mathrm{mM}$ of Hcy for $48 \mathrm{~h}$, while pretreatment with sirtinol $(15 \mu \mathrm{M}$, for $30 \mathrm{~min})$ increased the apoptosis in HT22 cells cotreated with Hcy and NaHS (Fig. 5C), which indiccated that sirtinol reverses the protection of NaHS against Hcy-induced apoptosis to HT22 cells. These results demonstrated that inhibition of SIRT1 reverses the protection of $\mathrm{H}_{2} \mathrm{~S}$ against Hcy-induced neurotoxicity in HT22 cells.

\section{Discussion}

Our previous studies have demonstrated that $\mathrm{H}_{2} \mathrm{~S}$ has a protective effect against Hcy-evoked neurotoxicity [20,24-26]. Considering the cellular senescence is prominent in the neurotoxicity of Hcy [3,27-29], the present work was designed to explore whether the protection of $\mathrm{H}_{2} \mathrm{~S}$ against the neurotoxicity of Hcy is associated with regulating neuronal senescence. The main findings of the present work are the following: (i) $\mathrm{H}_{2} \mathrm{~S}$ suppressed Hcy-induced cellular senescence in HT22; (ii) $\mathrm{H}_{2} \mathrm{~S}$ up-regulated the expression of SIRT1 in Hcy-exposed HT22 cells. (iii) Inhibition of SIRT1 reversed the protective effect of $\mathrm{H}_{2} \mathrm{~S}$ against Hcy-induced senescence and neurotoxicity in HT22 cells. Together, we demonstrated that the protection of $\mathrm{H}_{2} \mathrm{~S}$ against Hcy-induced neurotoxicity involves inhibition of neuronal senescence through upregulating SIRT1 signaling.

Cellular senescence, a process that imposes permanent proliferative arrest on cells in response to various stressors, has emerged as a potentially important contributor to aging and age-related disease, and it represents an attractive target for therapeutic exploitation [4]. Hcy is an independent risk factor for neurological and cardiovascular disease [30,31]. The level of Hcy in bodies 
increases with age [32]. Therefore, understanding whether Hcy induces neuronal senescence is necessary for a greater understanding of the neurotoxicity of Hcy, which may be part of the mechanisms leading to age-relate neurological diseases $[33,34]$. SA- $\beta-$ Gal staining remains as the most widely used biomarker for cellular senescence [35,36]. P21 CIPL is a cyclin-dependent kinase inhibitor, with regulating cell cycle progression at G1 and S phase [37]. P16 ${ }^{\text {INK4a }}$ plays a critical role on controlling cellular senescence [38]. The present work demonstrated that the percentage of SA- $\beta-G a l$ positive cells and the expressions of age-related markers P16 ${ }^{\mathrm{INK} K a}$ as well as P21 CIPL were significantly increased in Hcy-treated HT22 cells. Furthermore, we found that Hcy led to cell growth arrest. These data concluded that Hcy induces cellular senescence in HT22 cells. Therefore, modulation of neuronal senescence might represent a novel therapeutic strategy to overcome Hcy-induced neurotoxicity.

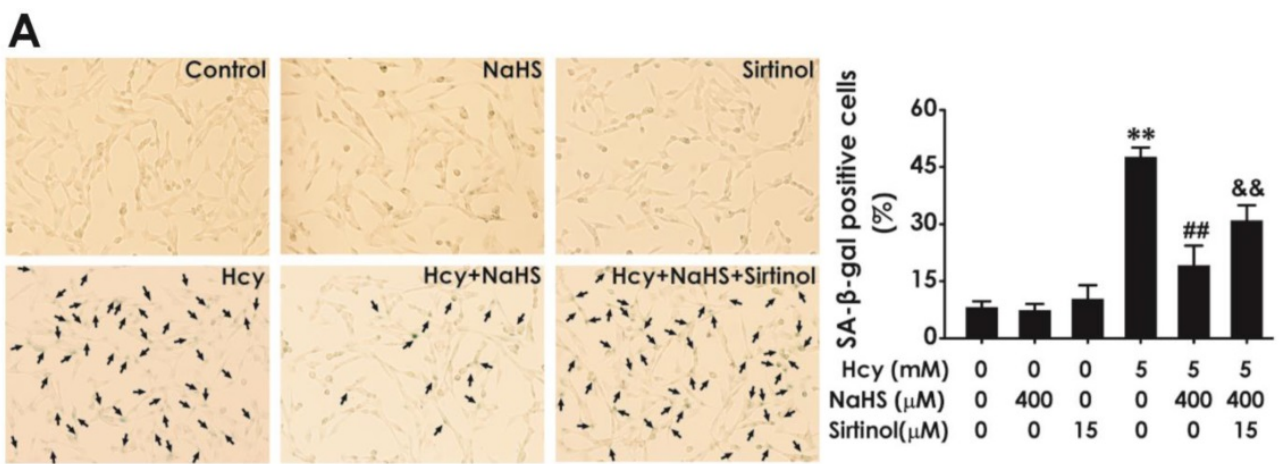

B
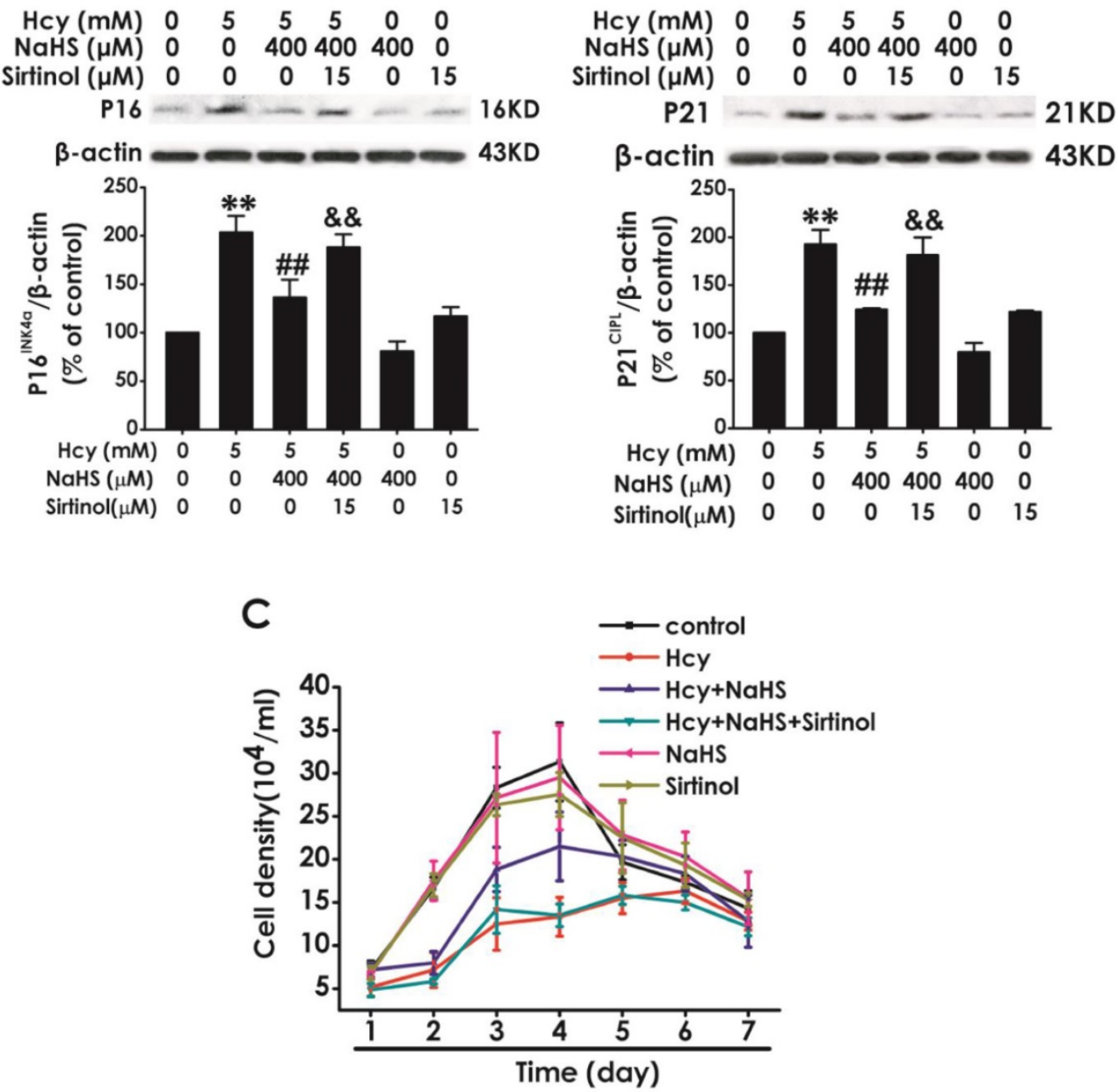

Figure 4. Effect of Sirtinol on NaHS-attenuated cellular senescence in Hcy-exposed HT22 cells. A, HT22 cells were stained with SA- $\beta$-gal and the SA- $\beta$-gal positive cell was quantitatively analyzed (magnification: $\times 10$; black arrows point the SA- $\beta$-gal staining positive cells). B, the expressions of P16INK4a and P21CIPL in HT22 cells were measured by western blotting. $C$, the cell density was determined by trypan blue analysis and the growth curve for $7 \mathrm{~d}$ was drawn. Values are mean $\pm \mathrm{SEM}(\mathrm{n}=3$ ). $* * P<0.01, v s$ control group; \#P<0.01, vs Hcy-treated alone group; \&P<0.05 \&\& $P<0.01$ vs cotreatment with Hcy and NaHS group. 
A

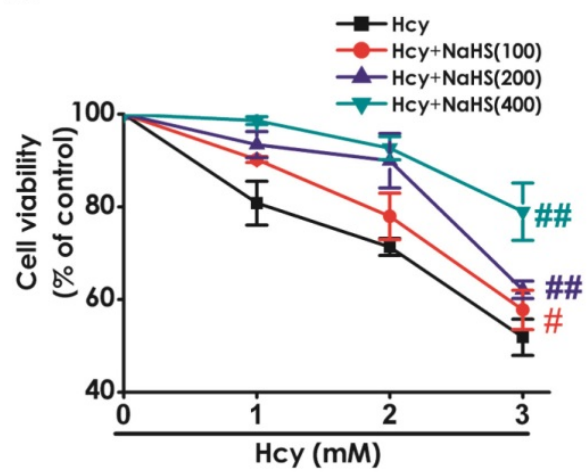

B

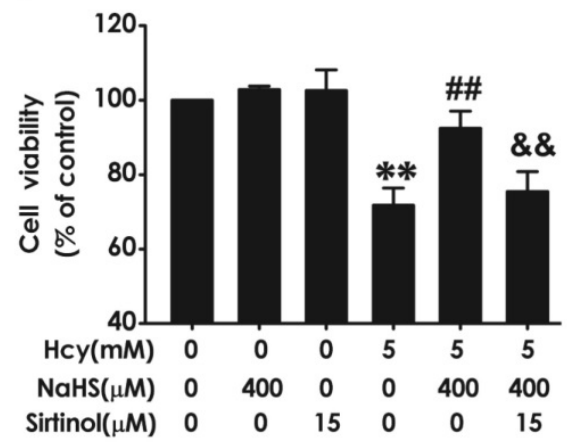

C
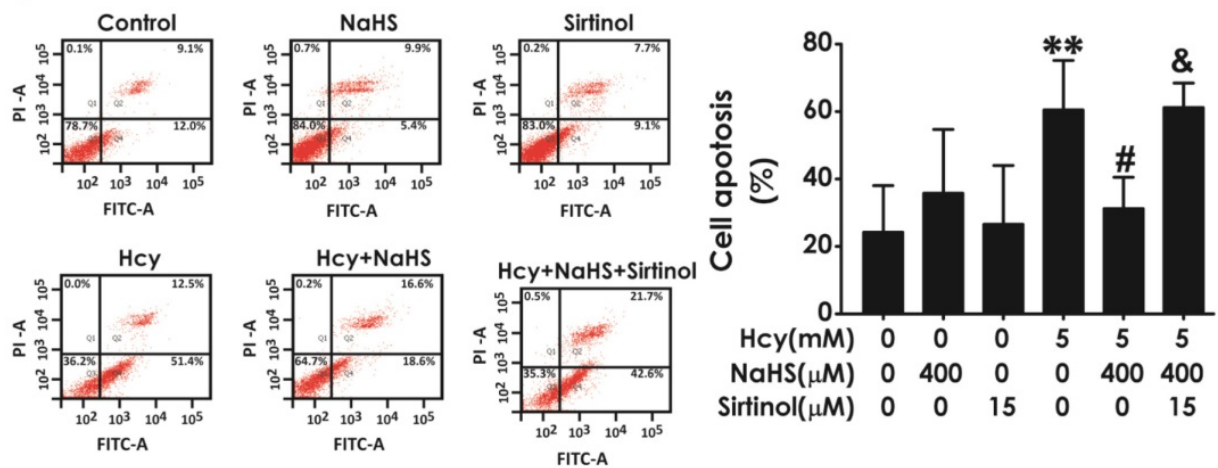

Figure 5. Effect of Sirtinol on the protection of $\mathbf{H}_{2} \mathbf{S}$ against $\mathbf{H c y}$-induced neurotoxicity to $\mathbf{H T 2 2}$ cells. A, the cells were treated with $2.5,5.0$, 10.0 mM Hcy, and 100 , $200,400 \mu \mathrm{mol} / \mathrm{L} \mathrm{NaHS}$ and routinely incubated for 48 hours. B-C, after preincubated with Sirtinol (15 mM) for 30 min, HT22 cells were pretreated with NaHS (400 uM) for 30 min, then cotreated with Hcy (5M) for $48 \mathrm{~h}$. A-B, the cell viability was determined by CCK-8 assay. C, the apoptosis of HT22 cells was assessed by flow cytometry after PI and Annexin $\mathrm{V}$ double staining (The annexin-V2/PI2 population is made up of normal healthy cells, while annexin- $\mathrm{V}+/ \mathrm{PI} 2$ cells exist in early apoptotic stage, and annexin- $\mathrm{V}+/ \mathrm{PI}+$ cells exist in late apoptotic stage). Values are the mean \pm SEM $(n=5)$. $* P<0.05 * * P<0.01$ vs control group; $\# P<0.05 \# P<0.01$ vs Hcy-treated alone group; \&P<0.05 vs cotreated with $\mathrm{NaHS}$ and Hcy group.

$\mathrm{H}_{2} \mathrm{~S}$, a well-known regulator of inflammation [39], ER stress [40] and cytotoxicity [41], is recognized as a new approach to prolong lifespan [12]. $\mathrm{H}_{2} \mathrm{~S}$ has been described to prevent endothelial cell senescence $[18,42]$ and delays aging [43]. Furthermore, we have previously found that $\mathrm{H}_{2} \mathrm{~S}$ inhibits formaldehydeinduced cellular senescence [44] and protects against Hcy-induced neurotoxicity [25]. Therefore, it is imperative to assess the effect of $\mathrm{H}_{2} \mathrm{~S}$ on Hcy-induced cellular senescence. In the present work, we found that $\mathrm{H}_{2} \mathrm{~S}$ increased the growth curve and decreased the percentage of SA- $\beta$-Gal positive cells as well as the expressions of P16 ${ }^{\text {INK4a }}$ and P21CIPL in Hcy-treated HT22 cells. Therefore, we for the first demonstrated that $\mathrm{H}_{2} \mathrm{~S}$ prevents Hcy-induced cellular senescence in HT22 cells. It has been confirmed that $\mathrm{H}_{2} \mathrm{~S}$ prevents the process of senescence in the endothelium [42], kidney [45,46], vascular [47], heart [48] and brain [43] of mice. Furthermore, increasing the content of endogenous $\mathrm{H}_{2} \mathrm{~S}$ by proper diet extends the life span of the aged mice $[49,50]$. These previous findings offered a reasonable explanation for the results obtained in the present study. Therefore, the regulation of neuronal senescence offers insights into the protection of $\mathrm{H}_{2} \mathrm{~S}$ against the neurotoxicity of Hcy. In our previous work, we used PC12 cells as a cell model to explore the protection of $\mathrm{H} 2 \mathrm{~S}$ against Hcy-induced endoplasmic reticulum (ER) stress [20]. This previous work focused on the protective role of $\mathrm{H}_{2} \mathrm{~S}$ in Parkinson's disease. Thus, we used PC12 cells to explore the protection of $\mathrm{H}_{2} \mathrm{~S}$ against Hcy-induced ER stress because PC12 cells are dopaminergic neurons and are commonly used as the cell model of PD. In the present study, the purpose is to explore the effect of $\mathrm{H}_{2} \mathrm{~S}$ on Hcy-induced impairment in learning and memory. Learning and memory are closely related to hippocampus [51]. Thus, HT22 cells were used in the present work because they are hippocampal neurons [52]. Hcy may be also induced ER stress in HT22 cells. In our future, we will explore the role of ER stress in Hcy-induced neuronal senescence in HT22 cells.

The present study also investigated the possible underlying mechanism for the protective role of $\mathrm{H}_{2} \mathrm{~S}$ in Hcy-induced senescence. Sirtuin was regarded as the lifespan-extending gene in the past years [53]. SIRT1, an important member of sirtuin family, also takes an important role in delaying cellular senescence and extending longevity [29,54]. Numerous studies have demonstrated that SIRT1 ameliorate neurodegenerative disease, such as $\mathrm{AD}$ [55], PD [56], subarachnoid hemorrhage [57], which 
reveal that SIRT1 delays senescence in the brain. Our previous study demonstrated that SIRT1 mediates the protective effect of $\mathrm{H}_{2} \mathrm{~S}$ on Hcy-induced neurotoxicity [20]. Therefore, we speculated whether SIRT1 mediates the protection of $\mathrm{H}_{2} \mathrm{~S}$ against Hcy-induced senescence in HT22 cells. How might Hcy mediate the blockade of SIRT1? Previous study suggests that Hcy increases ROS generation by activating protein kinase C- $\beta$ (PKC $\beta)$, which in turn improves SIRT1 degradation through a proteasome-dependent mechanism [58]. In line with this finding, our present work demonstrated that Hcy downregulated the expression of SIRT1 in HT22 cells. Notably, we found that $\mathrm{H}_{2} \mathrm{~S}$ not only increased the expressions of SIRT1 in HT22 cells, but also reversed Hcy-reduced the expressions of SIRT1 in HT22 cells. These results implied that the upregulation of SIRT1 contributed to the protective effect of $\mathrm{H}_{2} \mathrm{~S}$ on Hcy-induced senescence. To further confirm whether the SIRT1 mediates the protection of $\mathrm{H}_{2} \mathrm{~S}$ against Hcy-induced senescence, we explored whether the blockage of SIRT1 abolishes this protection of $\mathrm{H}_{2} \mathrm{~S}$. We utilized Sirtinol as the inhibitor of SIRT1, which get a broaden acceptance [59]. Our results showed that inhibited SIRT1 by Sirtinol abolished the protection of $\mathrm{H}_{2} \mathrm{~S}$ against Hcy-induced increase in the percentage of SA- $\beta$-Gal positive cells, up-regulations of P16 ${ }^{\mathrm{INK} 4 \mathrm{a}}$ and P21CIP1, and the arrest of cell growth. Taken together, these results indicated that SIRT1 mediates $\mathrm{H}_{2} \mathrm{~S}$-exerted protection against Hcy-induced senescence in HT-22 cells. Simultaneously, the blockage of SIRT1 also eliminated the protection of $\mathrm{H}_{2} \mathrm{~S}$ against Hcy-induced neurotoxicity, which further demonstrated that the involvement of reduced cellular senescence in the $\mathrm{H}_{2} \mathrm{~S}$-exerted protection against the neurotoxicity of Hcy. The possible molecular mechanism of $\mathrm{H}_{2} \mathrm{~S}$ action on SIRT1 is unknown. A recent study has described that $\mathrm{H}_{2} \mathrm{~S}$ increases intracellular NAD+ levels that are known to fuel SIRT1 activity [60] and that SIRT1 increases phosphorylation of extracellular signal-regulated protein kinases 1 and 2 (ERK1/2), which may be related to the regulation of anti-apoptosis and stress response [61,62]. Although the precise molecular mechanisms of H2S action on SIRT1 remain to be determined, the most promising candidates are $\mathrm{NAD}+$ or the pathway of PKC $\beta$-ROS. Future works will be required to precisely define whether NAD+ and ERK1/2 implicate in the mechanisms underlying the regulatory role of $\mathrm{H}_{2} \mathrm{~S}$ in the action of SIRT1.

\section{Conclusion}

In this study, we demonstrated that treatment with NaHS, a donor of $\mathrm{H}_{2} \mathrm{~S}$, attenuated Hcy-induced cellular senescence and upregulated SIRT1 expression in Hcy-exposed HT22 cells. Furthermore, we showed the reversing role of inhibited SIRT1 in the protection of NaHS against Hcy-induced cellular senescence and neurotoxicity. These data suggested that $\mathrm{H}_{2} \mathrm{~S}$ inhibits Hcy-induced cellular senescence by upregulation of SIRT1. Our results provide important insights into the molecular mechanism underlying $\mathrm{H}_{2} \mathrm{~S}$ exerted protection against Hcy-induced neurotoxicity and provide a basis for investigating $\mathrm{H}_{2} \mathrm{~S}$ as a therapeutic approach for Hcy-related neurodegenerative disease.

\section{Abbreviations}

Hcy: homocysteine; SIRT1: silence signal regulating factor $1 ; \mathrm{H}_{2} \mathrm{~S}$ : hydrogen sulfide; NaHS: sodium hydrosulfide; AD: Alzheimer's disease; PD: Parkinson's disease; DMSO: dimethyl sulfoxide; PI: propidium iodide; CCK-8: cell counting kit-8; DMEM: Dulbecco's Modified Eagle Medium; SA- $\beta-G a l$ : senescence-associated beta-galactosidase.

\section{Acknowledgements}

This study was supported by National Natural Science Foundation of China $(81671057,81771178)$ and Hunan Provincial Natural Science Foundation of China (2019JJ50546).

\section{Author Contributions}

Xiao-Qing Tang and Ke-Bin Zhan conceived and designed the study. Xuan Kang, Cheng Li, Xi Xie performed the experiments. San-Qiao Yang, Yi-Yun Tang, Wei Zou and Ping Zhang conducted the analysis. Xuan Kang and Cheng Li wrote the first version of the manuscript. Xiao-Qing Tang and Xuan Kang have maken necessary modifications to the manuscript. All authors have seen and approved the final manuscript.

\section{Competing Interests}

The authors have declared that no competing interest exists.

\section{References}

1 Prudova A, Bauman Z, Braun A et al. S-adenosylmethionine stabilizes cystathionine beta-synthase and modulates redox capacity. Proc Natl Acad Sci U S A. 2006; 103: 6489-6494.

2 Wei Z, Tiandong W, Yang L et al. Parkinson's Disease and Homocysteine: A Community-Based Study in a Folate and Vitamin B12 Deficient Population. Parkinsons Dis. 2016; 2016: 9539836.

3 McCully KS. Homocysteine Metabolism, Atherosclerosis, and Diseases of Aging. Compr Physiol. 2015; 6: 471-505.

4 Childs BG, Durik M, Baker DJ et al. Cellular senescence in aging and age-related disease: from mechanisms to therapy. Nat Med. 2015; 21: 1424-1435.

5 Campisi J \& d'Adda di Fagagna F. Cellular senescence: when bad things happen to good cells. Nat Rev Mol Cell Biol. 2007; 8: 729-740.

6 Childs BG, Gluscevic M, Baker DJ et al. Senescent cells: an emerging target for diseases of ageing. Nat Rev Drug Discov. 2017; 16: 718-735.

7 Zhang D, Sun X, Liu J et al. Homocysteine accelerates senescence of endothelial cells via DNA hypomethylation of human telomerase reverse transcriptase. Arterioscler Thromb Vasc Biol. 2015; 35: 71-78. 
8 Zhu JH, Chen JZ, Wang XX et al. Homocysteine accelerates senescence and reduces proliferation of endothelial progenitor cells. J Mol Cell Cardiol. 2006; 40: 648-652.

9 McCully KS. Review: Chemical Pathology of Homocysteine VI. Aging, Cellular Senescence, and Mitochondrial Dysfunction. Ann Clin Lab Sci. 2018; 48: 677-687.

10 Cao X, Cao L, Ding L et al. A New Hope for a Devastating Disease: Hydrogen Sulfide in Parkinson's Disease. Mol Neurobiol. 2018; 55: 3789-3799.

11 Xuan A, Long D, Li J et al. Hydrogen sulfide attenuates spatial memory impairment and hippocampal neuroinflammation in beta-amyloid rat model of Alzheimer's disease. J Neuroinflammation. 2012; 9: 202.

12 Qabazard B \& Sturzenbaum SR. H2S: A New Approach to Lifespan Enhancement and Healthy Ageing? Handb Exp Pharmacol. 2015; 230: 269-287.

13 Olson KR, Gao Y, Arif F et al. Metabolism of hydrogen sulfide (H2S) and Production of Reactive Sulfur Species (RSS) by superoxide dismutase. Redox Biol. 2018; 15: 74-85.

14 Yang G, Zhao K, Ju Y et al. Hydrogen sulfide protects against cellular senescence via S-sulfhydration of Keap1 and activation of Nrf2. Antioxid Redox Signal. 2013; 18: 1906-1919.

15 Xie ZZ, Shi MM, Xie L et al. Sulfhydration of p66Shc at cysteine59 mediates the antioxidant effect of hydrogen sulfide. Antioxid Redox Signal. 2014; 21: 2531-2542.

16 Zhao K, Ju Y, Li S et al. S-sulfhydration of MEK1 leads to PARP-1 activation and DNA damage repair. EMBO Rep. 2014; 15: 792-800.

17 Zheng $M$, Qiao $W$, Cui J et al. Hydrogen sulfide delays nicotinamide-induced premature senescence via upregulation of SIRT1 in human umbilical vein endothelial cells. Mol Cell Biochem. 2014; 393 : 59-67.

18 Suo R, Zhao ZZ, Tang $\mathrm{ZH}$ et al. Hydrogen sulfide prevents $\mathrm{H}(2) \mathrm{O}(2)$-induced senescence in human umbilical vein endothelial cells through SIRT1 activation. Mol Med Rep. 2013; 7: 1865-1870.

19 Tang XQ, Shen XT, Huang YE et al. Inhibition of endogenous hydrogen sulfide generation is associated with homocysteine-induced neurotoxicity: role of ERK1/2 activation. J Mol Neurosci. 2011; 45: 60-67.

20 Wang CY, Zou W, Liang XY et al. Hydrogen sulfide prevents homocysteineinduced endoplasmic reticulum stress in PC12 cells by upregulating SIRT1. Mol Med Rep. 2017; 16: 3587-3593.

21 Giblin W, Skinner ME \& Lombard DB. Sirtuins: guardians of mammalian healthspan. Trends in genetics : TIG. 2014; 30: 271-286.

22 Maiese K. New Insights for Oxidative Stress and Diabetes Mellitus. Oxid Med Cell Longev. 2015; 2015: 875961.

23 Fujitsuka N, Asakawa A, Morinaga A et al. Increased ghrelin signaling prolongs survival in mouse models of human aging through activation of sirtuin1. Mol Psychiatry. 2016; 21: 1613-1623.

24 Tang XQ, Shen XT, Huang YE et al. Hydrogen sulfide antagonizes homocysteine-induced neurotoxicity in PC12 cells. Neurosci Res. 2010; 68: 241-249.

25 Wei HJ, Xu JH, Li MH et al. Hydrogen sulfide inhibits homocysteine-induced endoplasmic reticulum stress and neuronal apoptosis in rat hippocampus via upregulation of the BDNF-TrkB pathway. Acta Pharmacol Sin. 2014; 35: 707-715.

$26 \mathrm{Li} \mathrm{M}$, Zhang $\mathrm{P}$, Wei $\mathrm{HJ}$ et al. Hydrogen Sulfide Ameliorates Homocysteine-Induced Cognitive Dysfunction by Inhibition of Reactive Aldehydes Involving Upregulation of ALDH2. Int J Neuropsychopharmacol. 2017; 20: 305-315.

27 Khuzakhmetova V, Yakovleva O, Dmitrieva S et al. Prenatal hyperhomocysteinemia induces oxidative stress and accelerates 'aging' of mammalian neuromuscular synapses. Int J Dev Neurosci. 2019; 75: 1-12.

28 Bossenmeyer-Pourie C, Smith AD, Lehmann S et al. N-homocysteinylation of tau and MAP1 is increased in autopsy specimens of Alzheimer's disease and vascular dementia. J Pathol. 2019; 248: 291-303.

29 Ostrakhovitch EA \& Tabibzadeh S. Homocysteine and age-associated disorders. Ageing Res Rev. 2019; 49: 144-164.

30 Rozycka A, Jagodzinski PP, Kozubski W et al. Homocysteine Level and Mechanisms of Injury in Parkinson's Disease as Related to MTHFR, MTR, and MTHFD1 Genes Polymorphisms and L-Dopa Treatment. Curr Genomics. 2013; 14: 534-542.

31 Bonetti F, Brombo G \& Zuliani G. The relationship between hyperhomocysteinemia and neurodegeneration. Neurodegener Dis Manag. 2016; 6: 133-145.

$32 \mathrm{Ma} \mathrm{F}, \mathrm{Wu}$ T, Zhao J et al. Plasma Homocysteine and Serum Folate and Vitamin B12 Levels in Mild Cognitive Impairment and Alzheimer's Disease: A Case-Control Study. Nutrients. 2017; 9.

33 Herrmann W \& Knapp JP. Hyperhomocysteinemia: a new risk factor for degenerative diseases. Clin Lab. 2002; 48: 471-481.
34 Hainsworth AH, Yeo NE, Weekman EM et al. Homocysteine, hyperhomocysteinemia and vascular contributions to cognitive impairment and dementia (VCID). Biochim Biophys Acta. 2016; 1862: 1008-1017.

35 Itahana K, Campisi J \& Dimri GP. Methods to detect biomarkers of cellular senescence: the senescence-associated beta-galactosidase assay. Methods Mol Biol. 2007; 371: 21-31.

36 Geng YQ, Guan JT, $\mathrm{Xu} \mathrm{XH}$ et al. Senescence-associated beta-galactosidase activity expression in aging hippocampal neurons. Biochem Biophys Res Commun. 2010; 396: 866-869.

37 Ma WD, Xu SR, Yan YL et al. Expressions of cyclin B1 and p21cipl in adult acute leukemia and their correlation. Zhongguo Shi Yan Xue Ye Xue Za Zhi. 2005; 13: 751-758.

38 Hall BM, Balan V, Gleiberman AS et al. p16(Ink4a) and senescence-associated beta-galactosidase can be induced in macrophages as part of a reversible response to physiological stimuli. Aging (Albany NY). 2017; 9: 1867-1884.

39 Bhatia M. H2S and Inflammation: An Overview. Handb Exp Pharmacol. 2015; 230: 165-180

40 George AK, Behera J, Kelly KE et al. Hydrogen sulfide, endoplasmic reticulum stress and alcohol mediated neurotoxicity. Brain Res Bull. 2017; 130: 251-256.

41 Guo R, Wu K, Chen J et al. Exogenous hydrogen sulfide protects against doxorubicin-induced inflammation and cytotoxicity by inhibiting p38MAPK/NFkappaB pathway in H9c2 cardiac cells. Cell Physiol Biochem. 2013; 32: 1668-1680.

42 Latorre E, Torregrossa R, Wood ME et al. Mitochondria-targeted hydrogen sulfide attenuates endothelial senescence by selective induction of splicing factors HNRNPD and SRSF2. Aging (Albany NY). 2018; 10: 1666-1681.

43 Zhan JQ, Zheng LL, Chen $\mathrm{HB}$ et al. Hydrogen Sulfide Reverses Aging-Associated Amygdalar Synaptic Plasticity and Fear Memory Deficits in Rats. Front Neurosci. 2018; 12: 390.

44 Zhu WW, Ning $M$, Peng $Y Z$ et al. Hydrogen Sulfide Inhibits Formaldehyde-Induced Senescence in HT-22 Cells via Upregulation of Leptin Signaling. Neuromolecular Med. 2019; 21: 192-203.

45 Hou CL, Wang MJ, Sun C et al. Protective Effects of Hydrogen Sulfide in the Ageing Kidney. Oxid Med Cell Longev. 2016; 2016: 7570489.

46 Lee HJ, Feliers D, Barnes JL et al. Hydrogen sulfide ameliorates aging-associated changes in the kidney. Geroscience. 2018; 40: 163-176.

47 Das A, Huang GX, Bonkowski MS et al. Impairment of an Endothelial $\mathrm{NAD}(+)-\mathrm{H} 2 \mathrm{~S}$ Signaling Network Is a Reversible Cause of Vascular Aging. Cell. 2018; 173: 74-89 e20.

48 Ma N, Liu HM, Xia T et al. Chronic aerobic exercise training alleviates myocardial fibrosis in aged rats through restoring bioavailability of hydrogen sulfide. Can J Physiol Pharmacol. 2018: 96: 902-908.

49 Yoshida S, Yamahara K, Kume S et al. Role of dietary amino acid balance in diet restriction-mediated lifespan extension, renoprotection, and muscle weakness in aged mice. Aging Cell. 2018; 17: e12796.

50 Hine C, Zhu Y, Hollenberg AN et al. Dietary and Endocrine Regulation of Endogenous Hydrogen Sulfide Production: Implications for Longevity. Antioxid Redox Signal. 2018; 28: 1483-1502.

51 Jarrard LE. On the role of the hippocampus in learning and memory in the rat. Behavioral and neural biology. 1993; 60: 9-26.

52 Chao XJ, Chen ZW, Liu AM et al. Effect of tacrine-3-caffeic acid, a novel multifunctional anti-Alzheimer's dimer, against oxidative-stress-induced cell death in HT22 hippocampal neurons: involvement of Nrf2/HO-1 pathway. CNS neuroscience \& therapeutics. 2014; 20: 840-850

53 Fujita Y \& Yamashita T. Sirtuins in Neuroendocrine Regulation and Neurological Diseases. Front Neurosci. 2018; 12: 778.

54 Ota $\mathrm{H}$, Akishita $\mathrm{M}$, Eto $\mathrm{M}$ et al. Sirt1 modulates premature senescence-like phenotype in human endothelial cells. J Mol Cell Cardiol. 2007; 43: 571-579.

55 Gomes BAQ, Silva JPB, Romeiro CFR et al. Neuroprotective Mechanisms of Resveratrol in Alzheimer's Disease: Role of SIRT1. Oxid Med Cell Longev. 2018; 2018: 8152373.

56 Singh P, Hanson PS \& Morris CM. SIRT1 ameliorates oxidative stress induced neural cell death and is down-regulated in Parkinson's disease. BMC Neurosci. 2017; 18: 46.

57 Zhang XS, Wu Q, Wu LY et al. Sirtuin 1 activation protects against early brain injury after experimental subarachnoid hemorrhage in rats. Cell Death Dis. 2016; 7: e2416.

58 Hung $\mathrm{CH}$, Chan $\mathrm{SH}$, Chu PM et al. Homocysteine facilitates LOX-1 activation and endothelial death through the PKCbeta and SIRT1/HSF1 mechanism: relevance to human hyperhomocysteinaemia. Clinical science. $2015 ;$ 129: 477-487. 
59 Kim MG, Kim DH, Lee HR et al. Sirtuin inhibition leads to autophagy and apoptosis in porcine preimplantation blastocysts. Biochem Biophys Res Commun. 2017; 488: 603-608.

60 Arumugam TV \& Kennedy BK. H2S to Mitigate Vascular Aging: A SIRT1 Connection. Cell. 2018; 173: 8-10.

61 Vauzour D, Vafeiadou K, Rice-Evans C et al. Activation of pro-survival Akt and ERK1/2 signalling pathways underlie the anti-apoptotic effects of flavanones in cortical neurons. Journal of neurochemistry. 2007; 103: 1355-1367.

62 Uchida S, Yamagata H, Seki T et al. Epigenetic mechanisms of major depression: Targeting neuronal plasticity. Psychiatry and clinical neurosciences. 2018; 72: 212-227. 